\title{
Shared Design Space: Sketching ideas using digital pens and a large augmented tabletop setup
}

\author{
Michael Haller ${ }^{1}$, Peter Brandl ${ }^{1}$, Daniel Leithinger ${ }^{1}$, Jakob Leitner ${ }^{1}$, Thomas Seifried ${ }^{1}$, \\ and Mark Billinghurst ${ }^{2}$ \\ 1 Digital Media, Upper Austria University of Applied Sciences, Austria \\ 2 HITLabNZ, University of Canterbury, New Zealand \\ coeno@fh-hagenberg. at
}

\begin{abstract}
Collaborative Augmented Reality (AR) setups are becoming increasingly popular. We have developed a collaborative tabletop environment that is designed for brainstorming and discussion meetings. Using a digital pen, participants can annotate not only virtual paper, but also real printouts. By integrating both forms of physical and digital paper, we combine virtual and real $2 \mathrm{~d}$ drawings, and digital data which are overlaid into a single information space. In this paper, we describe why we have integrated these devices together in a unique way and how they can be used efficiently during a design process.
\end{abstract}

\section{Introduction}

In recent years, Augmented Reality applications have been developed for many different platforms, such as mobile phones and handheld devices and also tabletop environments. Kiyokawa et al. describe the communication behaviors in a tabletop collaborative AR interface setup and they present several ways to improve the face-to-face collaboration by using AR [1]. In this paper, we describe a novel tabletop AR environment suitable for enhancing face to face collaboration, especially in the design process.

Designers and people who are discussing and brainstorming usually work in a studio surrounded with sketches, which are either pinned on a wall or placed on large surfaces. Currently, new sketches are mainly created directly on paper on the drafting table before developing a digital mock-up model on the computer. With new technology if may be possible to enhance this process. Blinn [2] postulates that the creative process is a two-phase process: firstly, moving from chaos to order and secondly, from ideation to implementation. Most computer-based design tools are primarily focussed on the second phase, and there is limited support for digital tools where people can play with ideas in a free form manner. Digital tabletop setups would be an ideal interface for sketching out a crude version of an idea. In the creative process, people still prefer using paper and large tables to capture their ideas. Therefore, the table still remains the main interaction device during the creative process. Augmented with virtual elements, a tabletop setup becomes an ideal input and output device around which people can share a wide range of verbal and non-verbal cues to collaborative effectively (cf. figure 1).

In this paper, we describe the combination of different hardware devices that can be combined to develop an AR-based tabletop environment for creating efficient applications [3]. Now, that it is technically possible to develop large augmented surfaces, it is 


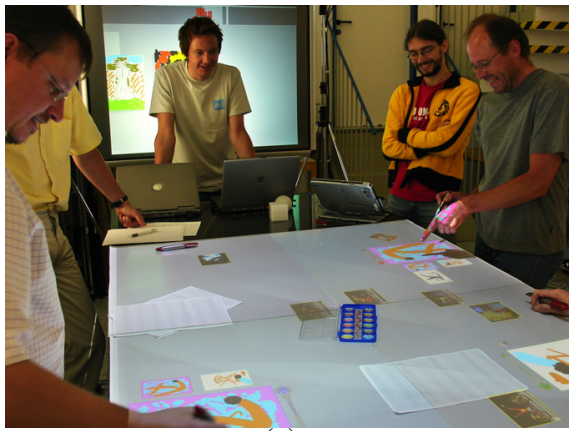

(a)

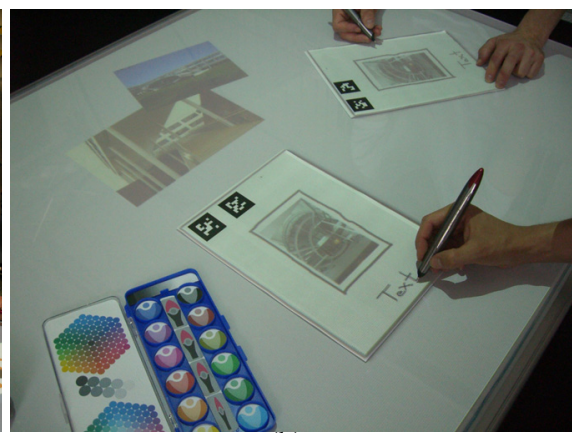

(b)

Fig. 1. (a) Participants can either interact with the interactive table either using tablet PCs or digital pens.(b) The collaboration mode allows to sketch the same document

important to conduct research on the different types of collaborative AR applications that are ideally suited for these types of tabletop setups and to present "user interface guidelines" for developing these applications. In the next section, we review related work on tabletop collaboration environments. Next, we present our system, focusing on hardware and design decisions. Section 3 focuses on interaction techniques. From the early user feedback, we conclude with design guidelines for collaborative tabletop AR systems and directions for future research.

\section{Related Work}

Early attempts at computer enhanced face-to-face collaboration involved conference rooms in which each participant had their own networked desktop computer that allowed them to send text or data to each other. However, these computer conference rooms were largely unsuccessful partly because of the lack of a common workspace [4]. An early improvement was using a video projector to provide a public display space. For example the Colab room at Xerox PARC [5] had an electronic whiteboard that any participate could use to display information to others. The importance of a central display for supporting face-to-face meetings has been recognized by the developers of large interactive commercial displays (such as the SMARTBoard DViT ). In traditional face-to-face conversation, people are able to equally contribute and interact with each other and with objects in the real world. However with large shared displays it is difficult to have equal collaboration when only one of the users has the input device, or the software doesn't support parallel input. In recent years, Stewart et al. coined the term Single Display Groupware (SDG) to describe groupware systems which support multiple input channels coupled to a single display [6]. They have found that SDG systems eliminate conflict among users for input devices, enable more work to be done in parallel by reducing turn-taking, and strengthen communication and collaboration. In general traditional desktop interface metaphors are less usable on large 
displays. For example, pull down menus may no longer be accessible, keyboard input may be difficult, and mouse input may require movement over large distances. A greater problem is that traditional desktop input devices do not allow people to use free-hand gesture or object-based interaction as they normally would in face-to-face collaboration. Researchers such as Ishii [7] have explored the use of tangible object interfaces for tabletop collaboration, while Streitz et al. [8] use natural gesture and object based interaction in their i-Land smart space. Regenbrecht et al. extend the idea of tangible user interfaces and demonstrates the benefits in novel video conference system [9]. In many interfaces there is a shared projected display visible by all participants; however, collaborative spaces can also support private data viewing. In Rekimoto's Augmented Surface's interface [10] users are able to bring their own laptop computers to a face-toface meeting and drag data from their private desktops onto a table or wall display area. They use an interaction technique called hyper-dragging which allows the projected display to become an extension of the user's personal desktop.

In the next section, we describe our AR tabletop system which combines these features. Unlike previous work, our system benefits from the following features:

- Seamless combination of both real and virtual data combined with augmented content,

- Intuitive data exchange using multiple (heterogeneous) devices based on natural and easy-to-use metaphors,

- Adapted and modified interaction methods (e.g. improved hyper-dragging for objects, Pick-and-Drop etc.), and

- Detailed discussion of what customers expect while using such a system. From the first meetings with our customers, we achieved a couple of interesting ideas which somehow diverge from the requirements seen by the developers.

\section{System Overview}

Our interface consists of four ceiling and a wall mounted projector showing data on a table surface (Interactive Table) and on an adjacent wall (Interactive Wall). All ceiling projectors are connected to a single display computer (cf. figure 2). Users can sit at the table and either connect their own laptop and/or tablet PC computer to the display server or interact directly with the table using digital pens. There is no limit as to how many clients can connect simultaneously to the system, and the amount of co-located participants depends on the space around the table. In our case, typically more than 5 participants are involved in a meeting, where one of the participants usually leads the session.

The tracking is realized by using large Anoto $^{3}$ patterns and digital pens from Maxell. Anoto-based pens are ballpoint-pens with an embedded camera that tracks the movements simultaneously (cf. figure 2). The pen has to be used on a specially printed paper with a pattern of tiny dots. Each paper sheet is unique and has its ID. The pen with its inbuilt infrared (IR) camera tracks the dots of the paper to retrieve both the ID of the paper and the position relative to the upper left corner of the paper. All required

\footnotetext{
${ }^{3}$ www.anoto.com
} 


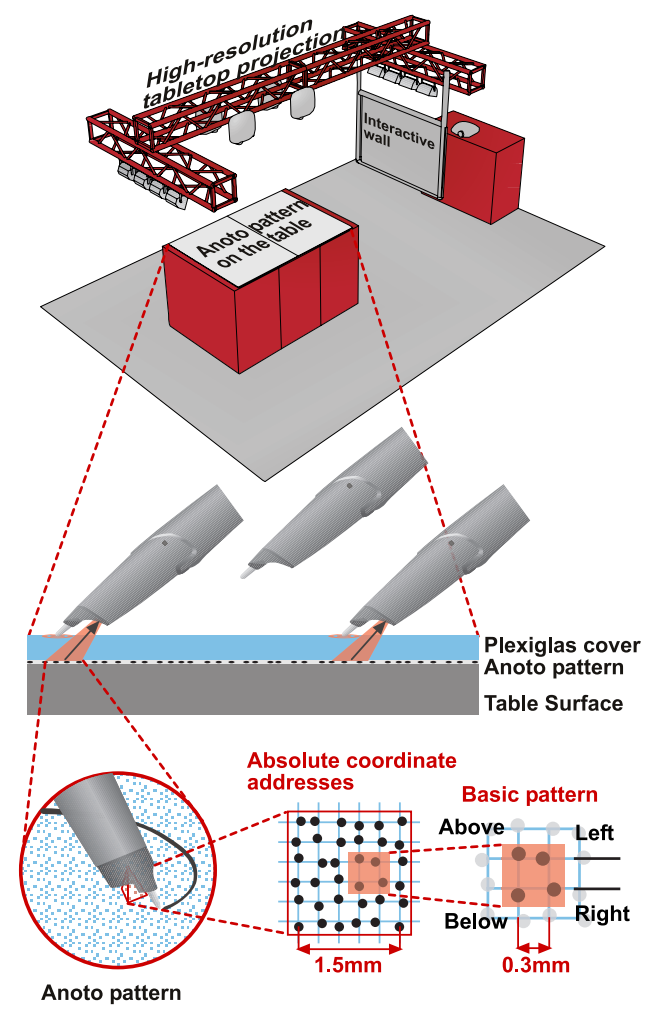

Fig. 2. The system consists of an interactive table and an interactive wall. The tracking on the table is realized using digital pens which can track the tiny dots of the Anoto pattern. To protect the Anoto pattern, we put a Plexiglas cover, which however, does not interfere the accurate tracking results.

informaion can then be sent in real-time to the PC using Bluetooth. Usually, the digital pens are used in combination with real printouts and real notebooks. However, we use this technology for tracking the users' movements and interactions on the whole table surface. To do this we put two A0-sized Anoto-patterns under the Plexiglas cover placed on the table (cf. figure 2). This allows us to track users' gesture input while holding the digital pen. Once the user touches the table with the pen, the camera tracks the underlying Anoto paper. Images projected onto to the table do not interfere with the tracking. Since every digital pen has its own personal ID, we can easily identify who is interacting with the interface without any additional hardware requirements (e.g. capacity measurement on the chairs [11]). Notice that the pens are also pressure sensitive which allows additional functionalities (i.e., for a better control in a sketching/drawing application).

We also allow real paper to be used in the interface. Real paper is socially wellaccepted in meetings and does not interfere with the face-to-face collaboration. In design meetings, participants often have to handle paper documents and drawings. Paper 
also has the advantage that it gives a fast and quick overview, provides a high resolution surface and is easy to carry. However, it can be difficult to transfer sketches on paper into digital applications. In our AR tabletop interface, we augment real paper with projected virtual graphics [12]. Participants can make annotations on the real content that is combined with digital content projected on top of the paper surface. The paper itself is tracked by using ARTag [13] markers, printed on each piece of paper.

Whenever a sketch is finished, it can be presented to the audience by moving it to the Interactive Wall. For the Interactive Wall setup we use a 60" diffuse Plexiglas display surface with special qualities for rear-projection. The anti-reflex surface homogeneously distributes the light to achieve a balanced image. A single camera tracking solution provides the interaction data for the sketch presentation. An IR pass filter that is applied to the camera avoids interferences with the projected image. As the tracking is done via shadows in the IR spectrum, IR lights are needed to provide a homogneous illumination in front of the screen. Touching the screen with one hand, the user can scroll through the sketches. A timeline on top provides a map-like overview that can also be used to navigate in a fast-forward style. Using both hands activates the zoom mode on screen, changing the distance between the hands affects the zoom-level accordingly. Notice that Anoto pens cannot be used on the interactive wall due to technical limitations: Althouth the embedded IR camera can track the graphite ink even on transparent surfaces (e.g. a pattern printed on a transparent foil), the rear-projected light interferes the tracking results.

\section{Interaction Techniques}

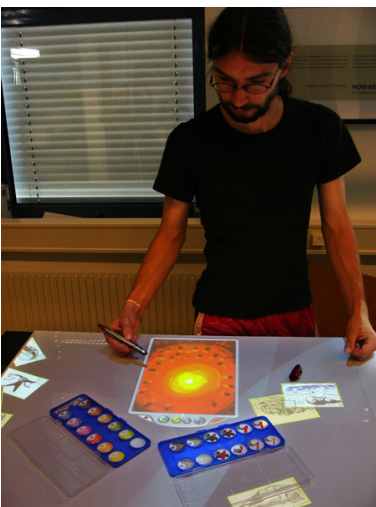

(a)

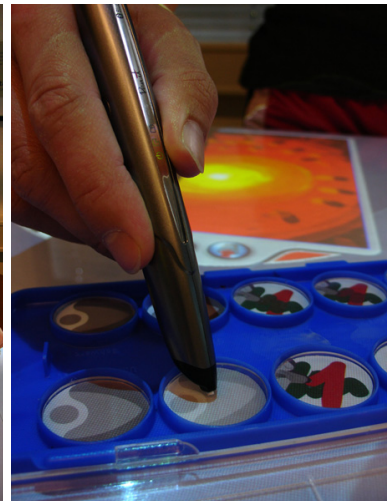

(b)

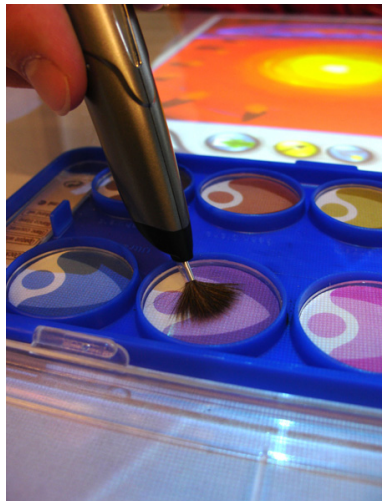

(c)

Fig. 3. Shared Design Space Interaction. (a) Designers can choose different color from a tangible interface (b) and sketch directly on the private workspace. (c) Optionally, users can also use pens with different caps. 
In this section, we mainly focus on novel interaction techniques we used in our project. We also present the different interaction possibilities and highlight why we have chosen the individual interface metaphors that we use. During a face-to-face brainstorming process, we can identify the following main tasks, which require adequate interaction techniques:

- Data Creation and Selection,

- Data Transfer, and

- Data Manipulation.

\subsection{Data Creation and Selection}

Usually, in the design process participants already have some sketches, notes, or drawings they want to share. On the tabletop system, users have two methods for data sharing, depending on the device they are using. If they have already some sketches on their private devices (e.g. tablet PC, laptop), the user can use traditional desktop tools for data creation. They can also create new sketches on the table using real/virtual paper and the digital pen. By observing users, we noticed that people preferred using the digital pen more; especially for sketching quick ideas. In fact, our system allows a seamless combination of digital and real content, where the augmented content has mainly be picked up from the table's surface. Thus, the table becomes a storage pool of digital content, where participants can share data. It is still a challenging task in which way the content initially comes onto the table. As mentioned before, we use additional devices, where the content can be moved smoothly to the table by hyper-dragging [10]. In the early phase, we did also experiments with a 1-degree of freedom (dof) menu, which was directly projected on the table surface (cf. figure 4). After drawing an ink-rectangle with

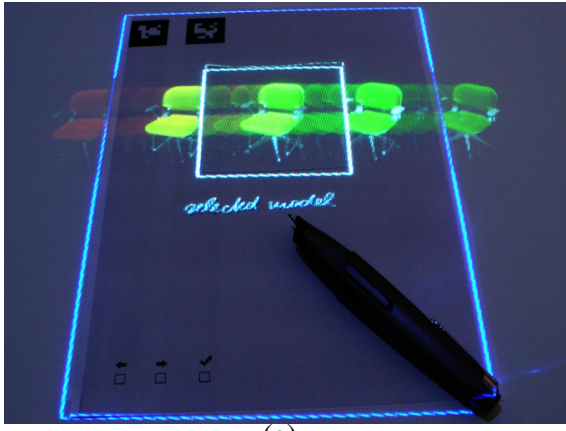

(a)

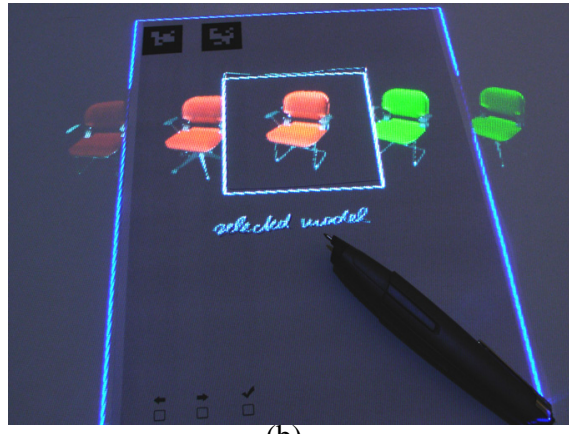

(b)

Fig. 4. 1-degree of freedom menu for selecting different $3 \mathrm{~d}$ geometries.

the pen, users get the 1-dof menu with additional digital content available. The user can interact by clicking with their pen on the special control elements (check boxes), which 
are printed on the real paper sheet. We used a default directory, which was browsed and shown in the menu. Once the corresponding geometry has been selected (again the user has to select the corresponding checkbox on the paper), the printout can be moved including the augmented content (e.g. videos). Notice that people can also select $3 \mathrm{~d}$ geometries simultaneously (cf. figure 4).

\subsection{Data Transfer}

In interfaces that contain multiple displays, an important research question is how to transfer data from one source to the other. In our setup, users sitting around the Interactive Table can move notes to the Interactive Wall by touching special control elements, projected onto the table surface and using the digital pen (cf. figure 5).

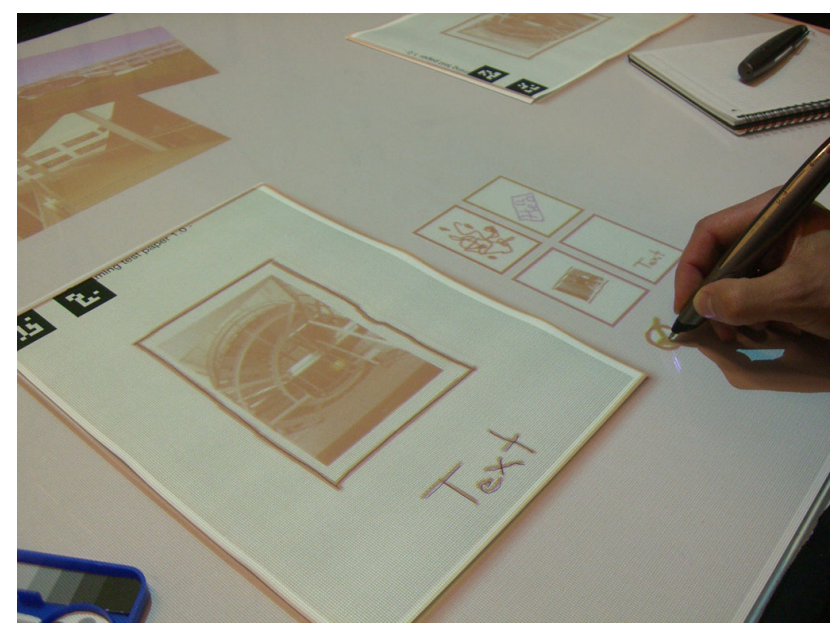

Fig. 5. Special control elements projected on the table surface allow to share the sketches, but also to transfer them to the Interactive Wall.

As noticed by Rekimoto in [14], people often have to transfer data from one device to another - especially in an augmented tabletop environment. In most cases, the traditional interaction metaphors (e.g. drag-and-drop) fail and more natural and quick data transfer metaphors have to be found.

In our setup, participants have three different possibilities of moving data from one screen to another depending on the device they are using (i.e. wireless presenter, personal device (e.g. laptop, tablet PC), and the Maxell pen).

\subsection{Moving Data with the Laptop PC (Hyper-Dragging)}

In our setup, all participants around the table can quickly re-arrange the content on the table using their personal devices and the user's mouse attached to their own devices. 
Therefore, users can click on a virtual image on their laptop computer desktop and drag it. Once the mouse reaches the edge of the physical display, the content appears on the table connected by a virtual line to the center of the desktop (cf. figure 6). Dragging with the mouse continues to move the note across the table.

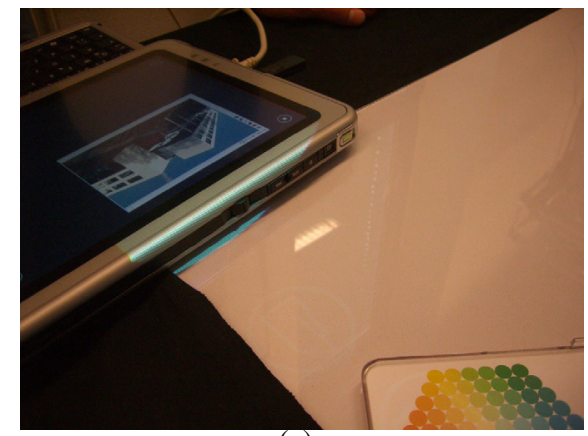

(a)

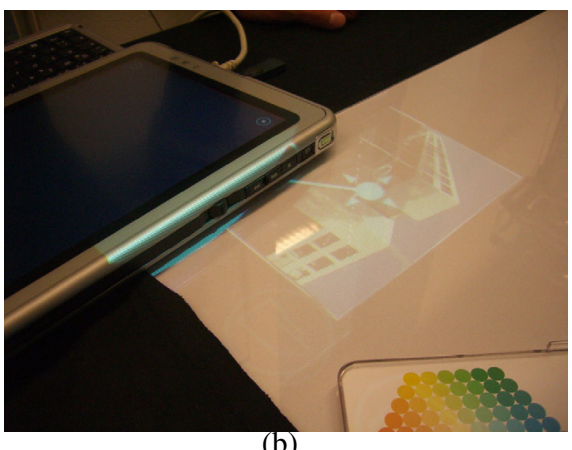

(b)

Fig. 6. Using the hyper-dragging metaphor allows an easy-to-use transfer of data between two devices (e.g. tablet PC and tabletop desk).

Participants can create new content on their private device and then move it to the public space. However, the direct interaction with content on the table surface is in many ways more natural that using mouse cursor input. In pilot studies people expected to be able to interact with their images once they were on the table surface and were surprised when this was initially not possible. We found that some people preferred using a device rather than interacting with their fingers. For this reason we added support for input from the Anoto digital pen.

\subsection{Moving Data with the Digital Pen (Pick-and-Drop)}

The combination of both real and virtual data requires a novel metaphor for data transfer. Instead of using the Drag-and-Drop metaphor, which is mainly used in 2d-based GUI applications, Rekimoto proposed the Pick-and-Drop metaphor [14]. It is more natural if users can manipulate and insert data in a computer environment as if it would be a real, physical object. In our setup, users can pick-up digital data from the table (e.g. video, images, $3 \mathrm{~d}$ geometry) and drop-it on the real paper by using the digital pen. Once the users pick an object, the digital pen tracks the underlying Anoto paper.

Once the user taps a digital object on the interactive table, our content manager automatically binds it (virtually) to the pen (cf. figure 7). Whenever the user moves the same pen onto the real paper, the manager transfers the data to the server that displays it on the interactive table. During our first tests, we recognized two reasons that the Pickand-Drop metaphor is more convenient than the Drag-and-Drop interaction. Firstly, our pens did not have additional buttons, which would have been necessary for realizing 


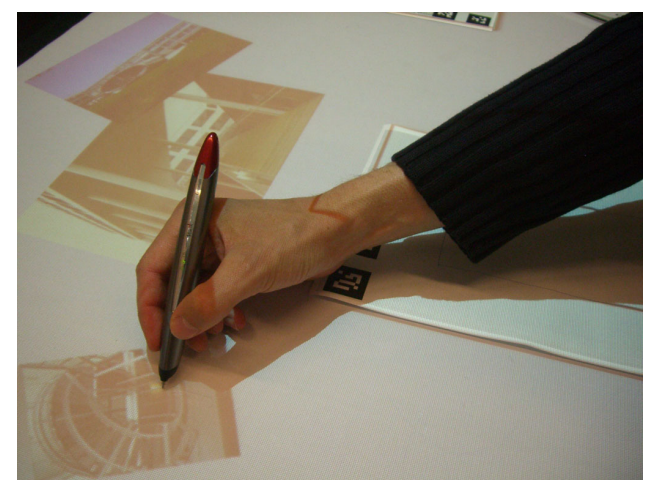

(a)

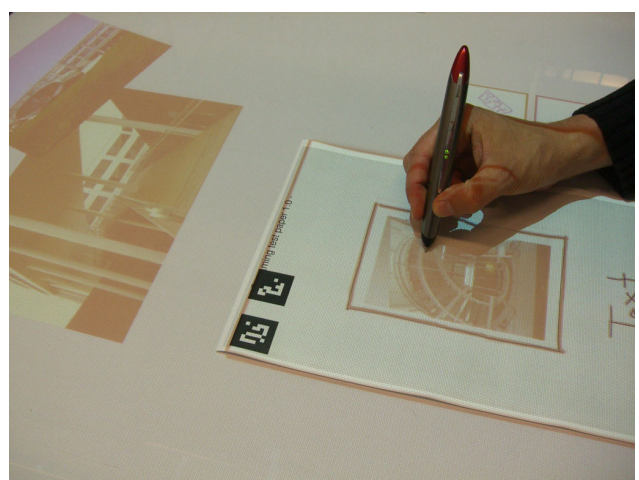

(b)

Fig. 7. Pick-and-Drop. Users can pick (a) an object from the interactive table to drop it (b) onto the real paper.

the Drag-and-Drop metaphor. Secondly, large movements on the table can be really uncomfortable and time-consuming, and are not required in the Pick-and-Drop metaphor. Robertson et al. presents improved Drag-and-Drop metaphors for wall-size displays for multiple data sets that could be integrated in the future [15].

\subsection{Moving Data on the Interactive Wall}

Once the digital data has been sent to the interactive wall, participants can move the data with users' gestures. We noticed during our first tests with end-users that the interactive wall is mainly used for presenting intermediate results. We also noticed that in most cases one person (mostly the coordinator of a session) stands up and presents the results to other participants.

\subsection{Data Manipulation}

On the interactive table, people can just move the sketches and perform simple transformations, such as translating, scaling, and rotating. Once participants want to manipulate the data sheet, they have to move it to private space (either to the personal tablet PC by hyper-dragging or to the personal workspace, as depicted in figure 3a).

An easy-to-use sketching tool allows the creation of simple strokes which are layered either onto the virtual data or onto the real printout (cf. [16][17]). The colors and brush strokes can be changed by using different tangible tools (e.g. the color chooser as depicted in figure 3). Again, the tiles of the color palette are printed with the special Anoto pattern. As mentioned before, users can also integrate virtual content (i.e., $2 \mathrm{~d}$ images, movies, or $3 \mathrm{~d}$ geometries) into the printout and/or the virtual paper. Not surprisingly, people also expect to have the possibility to transform the geometry or to start and/or stop a projected video. The ultimate goal would be that people draw their own control elements onto the paper and control the virtual content accordingly. 


\subsection{Group Interaction}

Using a collaborative interactive table means sharing information and working together. Often, in traditional meetings, people have neither enough hard-copies nor enough space for the large sketches (e.g. CAD sketches). Group interaction often becomes difficult and in many cases it is hard for user to see where their colleagues are pointing to. Moreover, the manual annotations (mainly done with markers or pens) cannot be stored digitally. Figure $1 \mathrm{~b}$ depicts a scenario where both users are working on the same paper sheet. All modifications are sent from the digital pen through Bluetooth to the server and the individuals' view of the projected comments is updated accordingly. The "shared desktop", which is embedded as a window in front of the collaborators, becomes a WYSIWIS (What You See Is What I See) window and all participants can see the same thing.The content control is mainly left to social norms. Discussing with participants, they have not felt the need to explicitly lock modification control over data objects, because they could always see who was attempting to modify their objects. However, it is important that users can identify who is manipulating each object (e.g. by using different colors).

\section{Implementation}

A system overview is presented in figure ??, which depicts the most important components. The system is written in $\mathrm{C}++$ based on OpenGL for rendering the virtual content and on ClanLib for rendering the graphical user interface. The advantage of the ClanLib library is that the system is based on top of OpenGL and can therefore be combined easily with the basic rendering system of the $3 \mathrm{~d}$ graphics library. In addition, we used the component model of the ClanLib library for the inter-component communication and the network extension for the communication between the clients and the server. The tracking of the markers attached on the printout was performed by the ARTag tracking library. The real-time tracking of the Maxell pen was implemented in C\# by using Maxell's Streaming API. Moreover, we use an in-house library for recognizing the axis-aligned rectangles drawn on the paper. The tracking on the rear-projection screen is mainly based on both the JazzUp library and OpenCV.

\section{Conclusions and Future Work}

In this paper, we presented the results of combining different easy-to-use interaction metaphors in a tabletop setup using digital pens and paper. Previous research demonstrated that AR technology can be used to enhance face-to-face collaboration in a natural way. The first results were strongly influenced by the first informal tests with our customers. The setup incorporates multiple devices and novel interaction metaphors to create an easy-to-use environment. The installation offers a cooperative and social experience by allowing multiple face-to-face participants to interact easily around a shared workspace, while also having access to their own private information space and a public presentation space combining both virtual and real sketches. The project is different 
from typical screen-based collaboration, because it uses advanced interface technologies to merge the person and task space. It is also uses multiple display surfaces to support private, group and public data viewing and interaction. The Anoto-based pen allows an ideal interface for interacting with both the real and virtual paper. Special control elements, projected on the desk, allow to share the own work for further discussion with the participants. Moreover, we included intuitive and natural objects (e.g. color palette etc.) and combined the real paper with digital content. We also investigated adequate and intuitive interaction metaphors for data movement (e.g. hyper-dragging, pick-and-drop etc.) and demonstrated their usage in the tabletop setup. We have also shown that the simultaneous use of the digital pen for the printout and for the interaction on the interactive table makes a lot of sense. The Anoto pattern used for tracking the users' movements seems to be an ideal configuration for similar tabletop applications.

Currently, we are starting with a formal evaluation. The main goal is to find out how the participants are using the real paper and in which sense they benefit from the augmented annotations. Finally, we also want to find out, in which sense a centralized data placement differs from a replicated note (cf. [18]). In a centralized design, participants only see one copy on the table. This often causes orientation and viewing problems by the participants who are sitting on the opposite of the table. Moreover, people have often problems, if one person is pointing to an object. To address these limitations, we also implemented the replicated view, where everybody can get the same view projected onto the personal paper sheet. Moreover, everybody can edit the paper simultaneously. In which sense traditional meetings will be influenced by a simultaneous interaction, will be investigated in a next user-study.

\section{Acknowledgements}

This research is funded in part by the Austrian FFG consortium (FHplus framework, no. 811407) and voestalpine Informationstechnologie. We also thank both Maxell and Anoto for their support. Finally, we thank our "Office of Tomorrow" team for providing inspirational ideas and constructive support for our work.

\section{References}

[1] Kiyokawa, K., Billinghurst, M., Hayes, S.E., Gupta, A., Sannohe, Y., Kato, H.: Communication behaviors of co-located users in collaborative ar interfaces. In: ISMAR '02: Proceedings of the International Symposium on Mixed and Augmented Reality (ISMAR'02), Washington, DC, USA, IEEE Computer Society (2002) 139

[2] Blinn, J.: The ultimate design tool. IEEE Computer Graphics and Applications 10(6) (1990) 90-92

[3] Haller, M., Billinghurst, M., Leithinger, D., Leitner, J., Seifried, T.: Coeno, enhancing faceto-face collaboration. In: ICAT '05: Proceedings of the 15th International Conference on Artificial Reality and Telexistence. (2005)

[4] Kori M. Inkpen, P.: Adapting the Human-Computer Interface to Support Collaborative Learning Environments for Children. PhD thesis, UBC (1997)

[5] Stefik, M., Foster, G., Bobrow, D.G., Kahn, K., Lanning, S., Suchman, L.: Beyond the chalkboard: computer support for collaboration and problem solving inmeetings (reprint). (1988) 335-366 
[6] Stewart, J., Bederson, B.B., Druin, A.: Single display groupware: A model for co-present collaboration. In: CHI. (1999) 286-293

[7] Ishii, H., Ullmer, B.: Tangible bits: Towards seamless interfaces between people, bits and atoms. In: Proceedings of CHI. (1997) 234-241

[8] Streitz, N., Tandler, P., Mller-Tomfelde, C., Konomi, S.: Roomware: Towards the next generation of human-computer interaction based on an integrated design of real and virtual worlds. In Carroll, J., ed.: Human-Computer Interaction in the New Millenium. AddisonWesley (2001) 553-578

[9] Regenbrecht, H., Lum, T., Kohler, P., Ott, C., Wagner, M., Wilke, W., Mueller, E.: Using augmented virtuality for remote collaboration. Presence: Teleoper. Virtual Environ. 13(3) (2004) 338-354

[10] Rekimoto, J., Saitoh, M.: Augmented surfaces: a spatially continuous work space for hybrid computing environments. In: CHI '99: Proceedings of the SIGCHI conference on Human factors in computing systems, New York, NY, USA, ACM Press (1999) 378-385

[11] Dietz, P., Leigh, D.: Diamondtouch: a multi-user touch technology. In: UIST'01: Proceedings of the 14th annual ACM symposium on User interface software and technology, New York, NY, USA, ACM Press (2001) 219-226

[12] Newman, W., Wellner, P.: A desk supporting computer-based interaction with paper documents. In: CHI '92: Proceedings of the SIGCHI conference on Human factors in computing systems, New York, NY, USA, ACM Press (1992) 587-592

[13] Fiala, M.: Artag, a fiducial marker system using digital techniques. In: CVPR '05: Proceedings of the 2005 IEEE Computer Society Conference on Computer Vision and Pattern Recognition (CVPR'05) - Volume 2, Washington, DC, USA, IEEE Computer Society (2005) 590-596

[14] Rekimoto, J.: Pick-and-drop: a direct manipulation technique for multiple computer environments. In: UIST '97: Proceedings of the 10th annual ACM symposium on User interface software and technology, New York, NY, USA, ACM Press (1997) 31-39

[15] Robertson, G., Czerwinski, M., Baudisch, P., Meyers, B., Robbins, D., Smith, G., Tan, D.: The large-display user experience. IEEE Comput. Graph. Appl. 25(4) (2005) 44-51

[16] Ishii, H., Ben-Joseph, E., Underkoffler, J., Yeung, L., Chak, D., Kanji, Z., Piper, B.: Augmented urban planning workbench: Overlaying drawings, physical models and digital simulation. ISMAR (2002) 203

[17] McGee, D.R., Cohen, P.R., Wu, L.: Something from nothing: augmenting a paper-based work practice via multimodal interaction. In: DARE '00: Proceedings of DARE 2000 on Designing augmented reality environments, New York, NY, USA, ACM Press (2000) 7180

[18] Morris, M.R., Paepcke, A., Winograd, T., Stamberger, J.: Teamtag: exploring centralized versus replicated controls for co-located tabletop groupware. In: CHI '06: Proceedings of the SIGCHI conference on Human Factors in computing systems, New York, NY, USA, ACM Press (2006) 1273-1282 